\title{
Implicit eigenvalue problems for maximal monotone operators
}

In-Sook Kim*

\author{
"Correspondence: iskim@skku.edu \\ Department of Mathematics, \\ Sungkyunkwan University, Suwon, \\ 440-746, Republic of Korea
}

\begin{abstract}
We study the implicit eigenvalue problem of the form

$0 \in T x+C(\lambda, x)$,

where $T$ is a maximal monotone multi-valued operator and the operator $C$ satisfies condition $\left(S_{+}\right)$or $\left(\tilde{S}_{+}\right)$. In a regularization method by the duality operator, we use the degree theories of Kartsatos and Skrypnik upon conditions of $C$ as well as Browder's degree. There are two cases to consider: One is that $C$ is demicontinuous and bounded with condition $\left(S_{+}\right)$; and the other is that $C$ is quasibounded and densely defined with condition $\left(\tilde{S}_{+}\right)$. Moreover, the eigenvalue problem $0 \in T x+\lambda C x$ is also discussed.
\end{abstract}

\section{Introduction and preliminaries}

A general eigenvalue theory for maximal monotone operators has been developed in various ways with applications to partial differential equations; see [5-7, 10-12]. A key tool was topological degrees for appropriate classes of operators in, e.g., $[2-4,8,9,14-16]$ and the method of approach was in many cases to use regularization by means of the duality operator, while in $[6,11]$ the eigenvalue problem was solved by a transformed equation in terms of the approximant without using the regularization method.

Let $X$ be a real reflexive Banach space with dual space $X^{*}$ and $T: D(T) \subset X \rightarrow 2^{X^{*}}$ be a maximal monotone multi-valued operator. When the resolvents of $T$ or the single-valued operator $C$ are compact, the solvability of the nonlinear inclusion

$$
0 \in T x+\lambda C x
$$

was studied in $[5,6,10,12]$ by applying the Leray-Schauder degree theory for compact operators. More generally, implicit eigenvalue problems were considered in $[7,10]$, where the single-valued and compact case was dealt with in [7]. In direction of [10], we study the implicit eigenvalue problem of the form

$$
0 \in T x+C(\lambda, x),
$$

() 2012 Kim; licensee Springer. This is an Open Access article distributed under the terms of the Creative Commons Attribution License (http://creativecommons.org/licenses/by/2.0), which permits unrestricted use, distribution, and reproduction in any medium, provided the original work is properly cited. 
where $C:[0, \Lambda] \times M \rightarrow X^{*}$ satisfies condition $\left(S_{+}\right)$or $\left(\tilde{S}_{+}\right)$. As in [12], we adopt property $(\mathcal{P})$ about the solvability of the related equation

$$
T_{s} x+C(\lambda, x)+\varepsilon J_{\psi} x=0
$$

where $T_{s}$ is the Brezis-Crandall-Pazy approximant introduced in [1] and $J_{\psi}$ is the (normalized) duality operator with a gauge function $\psi$.

In the present paper, we divide our investigation into two cases to apply suitable degree theory. One case deals with demicontinuous bounded operators satisfying condition $\left(S_{+}\right)$ with the aid of the most elementary degree theory of Skrypnik [14], in comparison with [10]. The other case is concerned with quasibounded densely defined operators satisfying condition $\left(\tilde{S}_{+}\right)$, where the degree theory of Kartsatos and Skrypnik $[8,9]$ for densely defined operators is used. In more concrete situations, the eigenvalue problem $0 \in T x+\lambda C x$ is discussed. We point out that Browder's degree in [3] for the reduced simple operator $T_{s}+\varepsilon J_{\psi}$ under the homotopy plays a crucial role in the proof of our results presented here.

Let $X$ be a real Banach space with its dual $X^{*}, \Omega$ a nonempty subset of $X$, and $Y$ another real Banach space. An operator $F: \Omega \rightarrow Y$ is said to be bounded if $F$ maps bounded subsets of $\Omega$ into bounded subsets of $Y$.F is said to be demicontinuous if for every $x_{0} \in \Omega$ and for every sequence $\left\{x_{n}\right\}$ in $\Omega$ with $x_{n} \rightarrow x_{0}$, we have $F x_{n} \rightarrow F x_{0}$. Here the symbol $\rightarrow(\rightarrow)$ stands for strong (weak) convergence.

A multi-valued operator $T: D(T) \subset X \rightarrow 2^{X^{*}}$ is said to be monotone if

$$
\left\langle u^{*}-v^{*}, x-y\right\rangle \geq 0 \quad \text { for every } x, y \in D(T) \text { and every } u^{*} \in T x, v^{*} \in T y \text {, }
$$

where $D(T)=\{x \in X: T x \neq \emptyset\}$ denotes the effective domain of $T$. $T$ is said to be maximal monotone if it is monotone and it follows from $\left(x, u^{*}\right) \in X \times X^{*}$ and

$$
\left\langle u^{\prime \prime}-v^{\prime \prime}, x-y\right\rangle \geq 0 \quad \text { for every } y \in D(T) \text { and every } v^{\prime \prime} \in T y
$$

that $x \in D(T)$ and $u^{*} \in T x$.

We say that an operator $T: D(T) \subset X \rightarrow X^{*}$ satisfies condition $\left(S_{+}\right)$if for every sequence $\left\{x_{n}\right\}$ in $D(T)$ with $x_{n} \rightarrow x_{0}$ and

$$
\limsup _{n \rightarrow \infty}\left\langle T x_{n}, x_{n}-x_{0}\right\rangle \leq 0,
$$

we have $x_{n} \rightarrow x_{0}$.

We say that $T: D(T) \subset X \rightarrow X^{*}$ satisfies condition $\left(\tilde{S}_{+}\right)$if for every sequence $\left\{x_{n}\right\}$ in $D(T)$ with $x_{n} \rightarrow x_{0}, T x_{n} \rightarrow h_{0}^{*}$ and

$$
\limsup _{n \rightarrow \infty}\left\langle T x_{n}, x_{n}-x_{0}\right\rangle \leq 0,
$$

we have $x_{n} \rightarrow x_{0}, x_{0} \in D(T)$ and $T x_{0}=h_{0}^{*}$.

We say that a multi-valued operator $T: D(T) \subset X \rightarrow 2^{X^{\circ}}$ satisfies condition $\left(S_{q}\right)$ on a set $M \subset D(T)$ if for every sequence $\left\{x_{n}\right\}$ in $M$ with $x_{n} \rightarrow x_{0}$ and every sequence $\left\{u_{n}^{*}\right\}$ in $X^{\prime \prime}$ with $u_{n}^{*} \rightarrow u^{*}$ where $u_{n}^{*} \in T x_{n}$, we have $x_{n} \rightarrow x_{0}$. 
Throughout this paper, $X$ will always be an infinite dimensional real reflexive Banach space which has been renormed so that $X$ and its dual $X^{*}$ are locally uniformly convex.

A function $\psi:[0, \infty) \rightarrow[0, \infty)$ is called a gauge function if $\psi$ is continuous, strictly increasing, $\psi(0)=0$ and $\psi(t) \rightarrow \infty$ as $t \rightarrow \infty$. An operator $J_{\psi}: X \rightarrow X^{*}$ is called a duality operator with a gauge function $\psi$ if

$$
\left\langle J_{\psi} x, x\right\rangle=\psi(\|x\|)\|x\| \quad \text { and } \quad\left\|J_{\psi} x\right\|=\psi(\|x\|) \quad \text { for } x \in X .
$$

If $\psi$ is the identity map $I$, then $J:=J_{I}$ is called a normalized duality operator. It is known in [13] that $J_{\psi}$ is continuous, bounded, surjective, strictly monotone, maximal monotone and satisfies condition $\left(S_{+}\right)$.

Given a maximal monotone operator $T: D(T) \subset X \rightarrow 2^{X^{*}}, x \in X$ and $s>0$, there exist unique elements $x_{s} \in D(T)$ and $u_{s}^{*} \in T x_{s}$ such that

$$
J\left(x_{s}-x\right)+s u_{s}^{*}=0 .
$$

Two operators $J_{s}: X \rightarrow D(T)$ and $T_{s}: X \rightarrow X^{*}$ defined by

$$
J_{s} x:=x_{s} \quad \text { and } \quad T_{s} x:=u_{s}^{*} \quad \text { for } x \in X
$$

are called the Brezis-Crandall-Pazy approximants. It is known in [1] that $J_{s}$ is continuous and bounded and $T_{s}$ is demicontinuous, bounded, and maximal monotone. It is easy to see that $J_{s}=I-s J^{-1} T_{s}$ and $T_{s} x \in T J_{s} x$ for $x \in X$.

Let $C:[0, \Lambda] \times M \rightarrow X^{*}$ be an operator, where $M$ is a subset of $X$. Then $C(t, x)$ is said to be continuous in $t$ uniformly with respect to $x \in M$ if for every $t_{0} \in[0, \Lambda]$ and for every sequence $\left\{t_{n}\right\}$ in $[0, \Lambda]$ with $t_{n} \rightarrow t_{0}$, we have $C\left(t_{n}, x\right) \rightarrow C\left(t_{0}, x\right)$ uniformly with respect to $x \in M$.

We say that $C$ satisfies condition $\left(S_{+}\right)$if for every $\lambda \in(0, \Lambda]$ and for every sequence $\left\{x_{n}\right\}$ in $M$ with $x_{n} \rightarrow x_{0}$ and

$$
\limsup _{n \rightarrow \infty}\left\langle C\left(\lambda, x_{n}\right), x_{n}-x_{0}\right\rangle \leq 0,
$$

we have $x_{n} \rightarrow x_{0}$.

We say that $C$ satisfies condition $\left(\tilde{S}_{+}\right)$if for every $\lambda \in(0, \Lambda]$ and for every sequence $\left\{x_{n}\right\}$ in $M$ with $x_{n} \rightarrow x_{0}, C\left(\lambda, x_{n}\right) \rightarrow h_{0}^{*}$ and

$$
\limsup _{n \rightarrow \infty}\left\langle C\left(\lambda, x_{n}\right), x_{n}-x_{0}\right\rangle \leq 0
$$

we have $x_{n} \rightarrow x_{0}, x_{0} \in M$ and $C\left(\lambda, x_{0}\right)=h_{0}^{*}$.

We often need the following demiclosedness property of maximal monotone operators given in [17].

Lemma 1.1 Let $T: D(T) \subset X \rightarrow 2^{X^{*}}$ be a maximal monotone multi-valued operator. Then for every sequence $\left\{x_{n}\right\}$ in $D(T), x_{n} \rightarrow x$ in $X$ and $u_{n}^{*} \rightarrow u^{\prime \prime}$ in $X^{\prime \prime}$, where $u_{n}^{\prime \prime} \in T x_{n}$, imply that $x \in D(T)$ and $u^{*} \in T x$. 


\section{Implicit eigenvalue problem about demicontinuous operators}

In this section, we are concerned with the implicit eigenvalue problem for perturbed maximal monotone operators in reflexive Banach spaces by applying the degree theories of Skrypnik and Browder for nonlinear operators of monotone type.

In what follows, for a bounded subset $\Omega$ of $X$, let $\bar{\Omega}$ and $\partial \Omega$ denote the closure and the boundary of $\Omega$ in $X$, respectively. Following Browder [2,3], a homotopy $H:[0,1] \times \bar{\Omega} \rightarrow X^{*}$ is said to be of class $\left(S_{+}\right)$if the following condition holds:

For every sequence $\left\{u_{j}\right\}$ in $\bar{\Omega}$ with $u_{j} \rightarrow u_{0}$ and every sequence $\left\{t_{j}\right\}$ in $[0,1]$ with $t_{j} \rightarrow t_{0}$ such that

$$
\limsup _{j \rightarrow \infty}\left\langle H\left(t_{j}, u_{j}\right), u_{j}-u_{0}\right\rangle \leq 0
$$

we have $u_{j} \rightarrow u_{0}$ and $H\left(t_{j}, u_{j}\right) \rightarrow H\left(t_{0}, u_{0}\right)$.

Kartsatos and Skrypnik [10] obtained the following result by using Browder's degree in [4] for multi-valued operators. As in [12], we adopt property $(\mathcal{P})$ in terms of the BrezisCrandall-Pazy approximant so that we can apply the most essential degree theory of Skrypnik [14] for single-valued operators to prove in a direct method.

Theorem 2.1 Let $\Omega$ be a bounded open set in $X$ with $0 \in \Omega$. Let $T: D(T) \subset X \rightarrow 2^{X^{*}}$ be a maximal monotone multi-valued operator with $0 \in D(T)$ and $0 \in T(0)$. Let $\Lambda$, $s_{0}$, and $\varepsilon_{0}$ be three positive numbers. Suppose that $C:[0, \Lambda] \times \bar{\Omega} \rightarrow X^{*}$ is demicontinuous, bounded and satisfies condition $\left(S_{+}\right)$such that $C(0, x)=0$ for all $x \in \bar{\Omega}$ and $C(t, x)$ is continuous in $t$ uniformly with respect to $x \in \bar{\Omega}$.

(a) For a given $\varepsilon>0$, assume the following property:

$(\mathcal{P})$ For every $s \in\left(0, s_{0}\right)$, there exists a $\lambda \in(0, \Lambda]$ such that the equation

$$
T_{s} x+C(\lambda, x)+\varepsilon J x=0
$$

has no solution in $\Omega$. Then there exists $a\left(\lambda_{\varepsilon}, x_{\varepsilon}\right) \in(0, \Lambda] \times(D(T) \cap \partial \Omega)$ such that

$$
0 \in T x_{\varepsilon}+C\left(\lambda_{\varepsilon}, x_{\varepsilon}\right)+\varepsilon J x_{\varepsilon} .
$$

(b) If $0 \notin T(D(T) \cap \partial \Omega)$, T satisfies condition $\left(S_{q}\right)$ on $D(T) \cap \partial \Omega$, and property $(\mathcal{P})$ is fulfilled for all $\varepsilon \in\left(0, \varepsilon_{0}\right]$, then there exists $a\left(\lambda_{0}, x_{0}\right) \in(0, \Lambda] \times(D(T) \cap \partial \Omega)$ such that

$$
0 \in T x_{0}+C\left(\lambda_{0}, x_{0}\right)
$$

Proof (a) We first claim that for any $s \in\left(0, s_{0}\right)$, there exists a $\left(\lambda_{0}, x_{0}\right) \in(0, \Lambda] \times \partial \Omega$ such that

$$
T_{s} x_{0}+C\left(\lambda_{0}, x_{0}\right)+\varepsilon J x_{0}=0 .
$$

Assume on the contrary that for some $s \in\left(0, s_{0}\right)$ and for every $\lambda \in(0, \Lambda]$, the following holds:

$$
T_{s} x+C(\lambda, x)+\varepsilon J x \neq 0 \quad \text { for all } x \in \partial \Omega .
$$


Since $\left(T_{s}+\varepsilon J\right)(0)=0$ and $T_{s}+\varepsilon J$ is injective by the strict monotonicity of $J$, we have $T_{s} x+$ $C(0, x)+\varepsilon J x \neq 0$ for all $x \in \partial \Omega$. Thus, (2.2) holds for all $\lambda \in[0, \Lambda]$.

Consider a homotopy $H:[0,1] \times \bar{\Omega} \rightarrow X^{*}$ defined by

$$
H(t, x)=T_{s} x+C(t \Lambda, x)+\varepsilon J x \quad \text { for }(t, x) \in[0,1] \times \bar{\Omega} .
$$

Then $H$ is of class $\left(S_{+}\right)$. To prove this, let $\left\{u_{j}\right\}$ be a sequence in $\bar{\Omega}$ with $u_{j} \rightarrow u_{0}$ and $\left\{t_{j}\right\}$ be a sequence in $[0,1]$ with $t_{j} \rightarrow t_{0}$ such that

$$
\limsup _{j \rightarrow \infty}\left\langle H\left(t_{j}, u_{j}\right), u_{j}-u_{0}\right\rangle \leq 0
$$

Since $T_{s}$ and $J$ are monotone, it follows from

$$
\left\langle H\left(t_{j}, u_{j}\right), u_{j}-u_{0}\right\rangle=\left\langle T_{s} u_{j}, u_{j}-u_{0}\right\rangle+\left\langle C\left(t_{j} \Lambda, u_{j}\right), u_{j}-u_{0}\right\rangle+\varepsilon\left\langle J u_{j}, u_{j}-u_{0}\right\rangle
$$

that

$$
\left\langle H\left(t_{j}, u_{j}\right), u_{j}-u_{0}\right\rangle \geq\left\langle T_{s} u_{0}, u_{j}-u_{0}\right\rangle+\left\langle C\left(t_{j} \Lambda, u_{j}\right), u_{j}-u_{0}\right\rangle+\varepsilon\left\langle J u_{j}, u_{j}-u_{0}\right\rangle
$$

and

$$
\left\langle H\left(t_{j}, u_{j}\right), u_{j}-u_{0}\right\rangle \geq\left\langle T_{s} u_{0}, u_{j}-u_{0}\right\rangle+\left\langle C\left(t_{j} \Lambda, u_{j}\right), u_{j}-u_{0}\right\rangle+\varepsilon\left\langle J u_{0}, u_{j}-u_{0}\right\rangle .
$$

By (2.3) and (2.5), we have

$$
\limsup _{j \rightarrow \infty}\left\langle C\left(t_{j} \Lambda, u_{j}\right), u_{j}-u_{0}\right\rangle \leq 0
$$

There are two cases to consider. If $t_{0}=0$, then $C\left(t_{j} \Lambda, u_{j}\right) \rightarrow 0$ and so

$$
\lim _{j \rightarrow \infty}\left\langle C\left(t_{j} \Lambda, u_{j}\right), u_{j}-u_{0}\right\rangle=0
$$

Using (2.3), (2.4), and (2.7), we obtain

$$
\limsup _{j \rightarrow \infty}\left\langle J u_{j}, u_{j}-u_{0}\right\rangle \leq 0
$$

Since $J$ satisfies condition $\left(S_{+}\right)$, we have

$$
u_{j} \rightarrow u_{0}
$$

which implies by the demicontinuity of $T_{s}$ and $C$ and the continuity of $J$

$$
T_{s} u_{j} \rightarrow T_{s} u_{0}, \quad C\left(t_{j} \Lambda, u_{j}\right) \rightarrow C\left(0, u_{0}\right), \quad \text { and } \quad J u_{j} \rightarrow J u_{0} .
$$

This means that $H\left(t_{j}, u_{j}\right) \rightarrow H\left(0, u_{0}\right)$. Now, let $t_{0}>0$. We have

$$
\left\langle C\left(t_{j} \Lambda, u_{j}\right), u_{j}-u_{0}\right\rangle=\left\langle C\left(t_{j} \Lambda, u_{j}\right)-C\left(t_{0} \Lambda, u_{j}\right), u_{j}-u_{0}\right\rangle+\left\langle C\left(t_{0} \Lambda, u_{j}\right), u_{j}-u_{0}\right\rangle
$$


which implies

$$
\begin{aligned}
& \limsup _{j \rightarrow \infty}\left\langle C\left(t_{0} \Lambda, u_{j}\right), u_{j}-u_{0}\right\rangle \\
& \quad \leq \limsup _{j \rightarrow \infty}\left\langle C\left(t_{j} \Lambda, u_{j}\right), u_{j}-u_{0}\right\rangle+\limsup _{j \rightarrow \infty}\left[-\left\langle C\left(t_{j} \Lambda, u_{j}\right)-C\left(t_{0} \Lambda, u_{j}\right), u_{j}-u_{0}\right\rangle\right]
\end{aligned}
$$

and hence by (2.6)

$$
\limsup _{j \rightarrow \infty}\left\langle C\left(t_{0} \Lambda, u_{j}\right), u_{j}-u_{0}\right\rangle \leq \lim _{j \rightarrow \infty}\left\|C\left(t_{j} \Lambda, u_{j}\right)-C\left(t_{0} \Lambda, u_{j}\right)\right\|\left\|u_{j}-u_{0}\right\|=0 .
$$

Since $C$ satisfies condition $\left(S_{+}\right)$, we get $u_{j} \rightarrow u_{0}$ from which $T_{s} u_{j} \rightarrow T_{s} u_{0}, C\left(t_{j} \Lambda, u_{j}\right) \rightarrow$ $C\left(t_{0} \Lambda, u_{0}\right)$, and $J u_{j} \rightarrow J u_{0}$. Consequently, $H\left(t_{j}, u_{j}\right) \rightarrow H\left(t_{0}, u_{0}\right)$. We have just shown that the homotopy $H$ is of class $\left(S_{+}\right)$.

We are now ready to apply the degree theory of Skrypnik $[14,15]$. If $d_{S}$ denotes the Skrypnik degree, the homotopy invariance property of $d_{S}$ implies that

$$
\begin{aligned}
d_{S}\left(T_{s}+C(\lambda, \cdot)+\varepsilon J, \Omega, 0\right) & =d_{S}(H(t, \cdot), \Omega, 0) \\
& =d_{S}\left(T_{s}+\varepsilon J, \Omega, 0\right) \\
& =1
\end{aligned}
$$

for all $\lambda \in[0, \Lambda]$. The last equality follows from Theorem 3 in [3], based on the fact that $T_{s}+$ $\varepsilon J$ is demicontinuous, bounded, injective, and satisfies condition $\left(S_{+}\right)$and $\left\langle T_{s} x+\varepsilon J x, x\right\rangle \geq 0$ for all $x \in \partial \Omega$. For every $\lambda \in(0, \Lambda]$, the existence property of $d_{S}$ implies that there is a point $x$ in $\Omega$ such that

$$
T_{s} x+C(\lambda, x)+\varepsilon J x=0
$$

which contradicts property $(\mathcal{P})$. Therefore, the first claim (2.1) is true.

In view of $(2.1)$, let $\left\{s_{n}\right\}$ be a sequence in $\left(0, s_{0}\right)$ with $s_{n} \rightarrow 0$ and $\left\{\left(\lambda_{n}, x_{n}\right)\right\}$ be the corresponding sequence in $(0, \Lambda] \times \partial \Omega$ such that

$$
T_{S_{n}} x_{n}+C\left(\lambda_{n}, x_{n}\right)+\varepsilon J x_{n}=0 .
$$

Without loss of generality, we may suppose that

$$
\lambda_{n} \rightarrow \lambda_{0}, \quad x_{n} \rightarrow x_{0}, \quad C\left(\lambda_{n}, x_{n}\right) \rightarrow c^{*} \quad \text { and } \quad J x_{n} \rightarrow j^{*},
$$

where $\lambda_{0} \in[0, \Lambda], x_{0} \in X, c^{*} \in X^{*}$ and $j^{*} \in X^{*}$. To arrive at the conclusion of (a) in the next step, we may suppose that $\lambda_{0} \in(0, \Lambda]$. In fact, when $\lambda_{0}=0$, we know that $0 \notin T x+C(0, x)+$ $\varepsilon J x$ for all $x \in D(T) \cap \partial \Omega$ because $T+\varepsilon J$ is injective on $D(T) \cap \bar{\Omega}$ and $0 \in(T+\varepsilon J)(D(T) \cap \Omega)$. For our aim, we will now show that

$$
\limsup _{n \rightarrow \infty}\left\langle C\left(\lambda_{n}, x_{n}\right), x_{n}-x_{0}\right\rangle \leq 0 .
$$


Assume on the contrary that there exists a subsequence of $\left\{x_{n}\right\}$, denoted again by $\left\{x_{n}\right\}$, such that

$$
\lim _{n \rightarrow \infty}\left\langle C\left(\lambda_{n}, x_{n}\right), x_{n}-x_{0}\right\rangle>0
$$

Note by (2.8) and the monotonicity of $J$ that

$$
\left\langle T_{s_{n}} x_{n}, x_{n}-x_{0}\right\rangle \leq-\left\langle C\left(\lambda_{n}, x_{n}\right), x_{n}-x_{0}\right\rangle-\varepsilon\left\langle J x_{0}, x_{n}-x_{0}\right\rangle
$$

and so

$$
\limsup _{n \rightarrow \infty}\left\langle T_{s_{n}} x_{n}, x_{n}-x_{0}\right\rangle<0
$$

Hence, it follows from $T_{S_{n}} x_{n} \rightarrow-c^{*}-\varepsilon j^{*}$ that

$$
\begin{aligned}
\limsup _{n \rightarrow \infty}\left\langle T_{S_{n}} x_{n}, x_{n}\right\rangle & =\limsup _{n \rightarrow \infty}\left[\left\langle T_{S_{n}} x_{n}, x_{n}-x_{0}\right\rangle+\left\langle T_{S_{n}} x_{n}, x_{0}\right\rangle\right] \\
& <\left\langle-c^{\prime \prime}-\varepsilon j^{\prime \prime}, x_{0}\right\rangle .
\end{aligned}
$$

Let $x \in D(T)$ and $u^{*} \in T x$ be arbitrary. Since $T$ is monotone, $T_{s_{n}} x_{n} \in T J_{s_{n}} x_{n}$, and $J_{s_{n}}=$ $I-s_{n} J^{-1} T_{s_{n}}$, we have

$$
\left\langle T_{s_{n}} x_{n}-u^{\prime \prime}, x_{n}-s_{n} J^{-1} T_{s_{n}} x_{n}-x\right\rangle \geq 0,
$$

which implies

$$
\left\langle T_{s_{n}} x_{n}, x_{n}\right\rangle \geq s_{n}\left\|T_{s_{n}} x_{n}\right\|^{2}+\left\langle T_{s_{n}} x_{n}, x\right\rangle+\left\langle u^{*}, x_{n}-x\right\rangle-s_{n}\left\langle u^{\prime \prime}, J^{-1} T_{s_{n}} x_{n}\right\rangle
$$

Since $\left\{T_{s_{n}} x_{n}\right\}$ and $J^{-1}$ are bounded and $s_{n} \rightarrow 0$, we have

$$
\liminf _{n \rightarrow \infty}\left\langle T_{S_{n}} x_{n}, x_{n}\right\rangle \geq\left\langle-c^{*}-\varepsilon j^{*}, x\right\rangle+\left\langle u^{*}, x_{0}-x\right\rangle
$$

Combining (2.10) and (2.11), we get

$$
\left\langle-c^{*}-\varepsilon j^{*}-u^{*}, x_{0}-x\right\rangle>0 \quad \text { for all } x \in D(T) \text { and } u^{*} \in T x \text {. }
$$

Since $T$ is maximal monotone, we have $x_{0} \in D(T)$ and $-c^{*}-\varepsilon j^{*} \in T x_{0}$. Letting $x=x_{0} \in D(T)$ in (2.12) yields a contradiction. Thus, (2.9) holds.

Since $C(t, x)$ is continuous in $t$ uniformly with respect to $x \in \bar{\Omega}$ and $\lambda_{n} \rightarrow \lambda_{0}$, we have by (2.9)

$$
\limsup _{n \rightarrow \infty}\left\langle C\left(\lambda_{0}, x_{n}\right), x_{n}-x_{0}\right\rangle \leq 0,
$$

by observing that

$$
\left\langle C\left(\lambda_{n}, x_{n}\right), x_{n}-x_{0}\right\rangle=\left\langle C\left(\lambda_{n}, x_{n}\right)-C\left(\lambda_{0}, x_{n}\right), x_{n}-x_{0}\right\rangle+\left\langle C\left(\lambda_{0}, x_{n}\right), x_{n}-x_{0}\right\rangle .
$$


Since $C$ satisfies condition $\left(S_{+}\right)$, it follows from (2.13) that

$$
x_{n} \rightarrow x_{0} \in \partial \Omega
$$

which implies by $(2.8)$

$$
T_{s_{n}} x_{n} \rightarrow-C\left(\lambda_{0}, x_{0}\right)-\varepsilon J x_{0}
$$

and as above

$$
J_{s_{n}} x_{n}=x_{n}-s_{n} J^{-1} T_{s_{n}} x_{n} \rightarrow x_{0} .
$$

Since $T$ is maximal monotone and $T_{s_{n}} x_{n} \in T J_{s_{n}} x_{n}$, Lemma 1.1 implies that $x_{0} \in D(T)$ and

$$
0 \in T x_{0}+C\left(\lambda_{0}, x_{0}\right)+\varepsilon J x_{0} .
$$

(b) According to the statement (a), for a sequence $\left\{\varepsilon_{n}\right\}$ in $\left(0, \varepsilon_{0}\right]$ with $\varepsilon_{n} \rightarrow 0$, we can choose sequences $\left\{\lambda_{n}\right\}$ in $(0, \Lambda],\left\{x_{n}\right\}$ in $D(T) \cap \partial \Omega$, and $\left\{u_{n}^{*}\right\}$ in $X^{*}$ with $u_{n}^{*} \in T x_{n}$ such that

$$
u_{n}^{*}+C\left(\lambda_{n}, x_{n}\right)+\varepsilon_{n} J x_{n}=0 .
$$

We may suppose that

$$
\lambda_{n} \rightarrow \lambda_{0}, \quad x_{n} \rightarrow x_{0}, \quad C\left(\lambda_{n}, x_{n}\right) \rightarrow c^{*} \quad \text { and } J x_{n} \rightarrow j^{*},
$$

where $\lambda_{0} \in[0, \Lambda], x_{0} \in X, c^{*} \in X^{*}$, and $j^{*} \in X^{*}$. Note that $\lambda_{0} \in(0, \Lambda]$. Indeed, if $\lambda_{0}=0$, then (2.14) implies $u_{n}^{*} \rightarrow 0$ which gives by condition $\left(S_{q}\right)$ on $D(T) \cap \partial \Omega, x_{n} \rightarrow x_{0} \in \partial \Omega$ and therefore $x_{0} \in D(T)$ and $0 \in T x_{0}$, which contradicts the hypothesis that $0 \notin T(D(T) \cap$ $\partial \Omega)$

To show that $x_{n} \rightarrow x_{0}$, we first claim that

$$
\limsup _{n \rightarrow \infty}\left\langle C\left(\lambda_{n}, x_{n}\right), x_{n}-x_{0}\right\rangle \leq 0
$$

Assume the contrary. So, we may suppose that

$$
\lim _{n \rightarrow \infty}\left\langle C\left(\lambda_{n}, x_{n}\right), x_{n}-x_{0}\right\rangle>0
$$

Hence, it follows from (2.14) that $u_{n}^{*} \rightarrow-c^{*}$ and

$$
\limsup _{n \rightarrow \infty}\left\langle u_{n}^{*}, x_{n}-x_{0}\right\rangle<0,
$$

which imply

$$
\limsup _{n \rightarrow \infty}\left\langle u_{n}^{*}, x_{n}\right\rangle<\left\langle-c^{*}, x_{0}\right\rangle .
$$


For every $x \in D(T)$ and every $u^{*} \in T x$, we obtain from the monotonicity of $T$ that

$$
\begin{aligned}
\liminf _{n \rightarrow \infty}\left\langle u_{n}^{*}, x_{n}\right\rangle & \geq \liminf _{n \rightarrow \infty}\left[\left\langle u_{n}^{*}, x\right\rangle+\left\langle u^{*}, x_{n}-x\right\rangle\right] \\
& \geq\left\langle-c^{*}, x\right\rangle+\left\langle u^{*}, x_{0}-x\right\rangle,
\end{aligned}
$$

which implies along with (2.16)

$$
\left\langle-c^{*}-u^{*}, x_{0}-x\right\rangle>0
$$

Since $T$ is maximal monotone, we have $x_{0} \in D(T)$ and $-c^{*} \in T x_{0}$. Letting $x=x_{0}$ in (2.17), we have a contradiction. Thus, (2.15) is true.

As above, we can deduce from (2.15) that

$$
\limsup _{n \rightarrow \infty}\left\langle C\left(\lambda_{0}, x_{n}\right), x_{n}-x_{0}\right\rangle \leq 0
$$

Since $C$ satisfies condition $\left(S_{+}\right)$and is demicontinuous, we obtain from (2.14) that

$$
x_{n} \rightarrow x_{0} \text { and } u_{n}^{*} \rightarrow-C\left(\lambda_{0}, x_{0}\right) \text {. }
$$

We conclude that $x_{0} \in D(T) \cap \partial \Omega$ and

$$
0 \in T x_{0}+C\left(\lambda_{0}, x_{0}\right)
$$

This completes the proof.

As a consequence of Theorem 2.1, we have the following result. When $C$ is a compact operator, it was proved by Li and Huang [12] with the aid of the Leray-Schauder degree for compact operators.

Corollary 2.2 Let $T, \Omega, \Lambda, s_{0}$, and $\varepsilon_{0}$ be as in Theorem 2.1. Suppose that $C: \bar{\Omega} \rightarrow X^{*}$ is a demicontinuous bounded operator which satisfies condition $\left(S_{+}\right)$.

(a) For a given $\varepsilon>0$, assume the following property:

$(\mathcal{P})$ For every $s \in\left(0, s_{0}\right)$, there exists a $\lambda \in(0, \Lambda]$ such that the equation

$$
T_{s} x+\lambda C x+\varepsilon J x=0
$$

has no solution in $\Omega$. Then there exists $a\left(\lambda_{\varepsilon}, x_{\varepsilon}\right) \in(0, \Lambda] \times(D(T) \cap \partial \Omega)$ such that

$$
0 \in T x_{\varepsilon}+\lambda_{\varepsilon} C x_{\varepsilon}+\varepsilon J x_{\varepsilon} .
$$

(b) If $0 \notin T(D(T) \cap \partial \Omega)$, T satisfies condition $\left(S_{q}\right)$ on $D(T) \cap \partial \Omega$, and property $(\mathcal{P})$ is fulfilled for all $\varepsilon \in\left(0, \varepsilon_{0}\right]$, then there exists $a\left(\lambda_{0}, x_{0}\right) \in(0, \Lambda] \times(D(T) \cap \partial \Omega)$ such that

$$
0 \in T x_{0}+\lambda_{0} C x_{0}
$$


Proof Define an operator $\tilde{C}:[0, \Lambda] \times \bar{\Omega} \rightarrow X^{*}$ by

$$
\tilde{C}(\lambda, x)=\lambda C x \quad \text { for }(\lambda, x) \in[0, \Lambda] \times \bar{\Omega} .
$$

By hypotheses on $C$, the operator $\tilde{C}$ is obviously demicontinuous, bounded, and satisfies condition $\left(S_{+}\right)$. Moreover, $\tilde{C}(t, x)$ is continuous in $t$ uniformly with respect to $x \in \bar{\Omega}$ because $C(\bar{\Omega})$ is bounded. Apply Theorem 2.1 with $C=\tilde{C}$.

\section{Implicit eigenvalue problem about densely defined operators}

In this section, we study the implicit eigenvalue problem for densely defined perturbations of maximal monotone operators, based on the degree theories of Kartsatos and Skrypnik.

An operator $C:[0, \Lambda] \times D(C) \rightarrow X^{*}$ is said to be uniformly quasibounded if for every $S>0$ there exists a constant $K(S)>0$ such that for all $\lambda \in[0, \Lambda]$ and all $u \in D(C)$ with $\|u\| \leq S$ and $\langle C(\lambda, u), u\rangle \leq 0$, we have $\|C(\lambda, u)\| \leq K(S)$.

In a regularization method by the duality operator, we establish a new result on the existence of eigenvalues, by applying topological degree for densely defined operators in $[8,9]$.

Theorem 3.1 Let $\Omega$ be a bounded open set in $X$ with $0 \in \Omega$ and $L$ a dense subspace of $X$. Let $T: D(T) \subset X \rightarrow 2^{X^{*}}$ be a maximal monotone multi-valued operator with $0 \in D(T)$ and $0 \in T(0)$. Let $\Lambda, s_{0}$, and $\varepsilon_{0}$ be positive numbers. Assume that $C:[0, \Lambda] \times D(C) \rightarrow X^{*}$ is a single-valued operator with $L \subset D(C) \subset X$ such that $C(0, x)=0$ for all $x \in D(C)$ and $C(t, x)$ is continuous in $t$ uniformly with respect to $x \in D(C)$. Assume further that

(c1) $C$ is uniformly quasibounded,

(c2) C satisfies condition $\left(\tilde{S}_{+}\right)$, and

(c3) for every $\lambda \in[0, \Lambda]$ and for every $F \in \mathcal{F}(L)$ and $v \in L$, the function $c(\lambda, F, v): F \rightarrow \mathbb{R}$, $c(\lambda, F, v)(u)=\langle C(\lambda, u), v\rangle$, is continuous on $F$, where $\mathcal{F}(L)$ denotes the set of all finite-dimensional subspaces of $L$.

Then the following statements hold:

(a) For a given $\varepsilon>0$, assume the following property:

$(\mathcal{P})$ For every $s \in\left(0, s_{0}\right)$, there exists a $\lambda \in(0, \Lambda]$ such that the equation

$$
T_{s} x+C(\lambda, x)+\varepsilon J_{\psi} x=0
$$

has no solution in $D(C) \cap \Omega$. Then there is a $\left(\lambda_{\varepsilon}, x_{\varepsilon}\right) \in(0, \Lambda] \times(D(T) \cap D(C) \cap \partial \Omega)$ such that

$$
0 \in T x_{\varepsilon}+C\left(\lambda_{\varepsilon}, x_{\varepsilon}\right)+\varepsilon J_{\psi} x_{\varepsilon}
$$

(b) If $0 \notin T(D(T) \cap \partial \Omega)$, $T$ satisfies condition $\left(S_{q}\right)$ on $D(T) \cap \partial \Omega$, and property $(\mathcal{P})$ is fulfilled for all $\varepsilon \in\left(0, \varepsilon_{0}\right]$, then there exists a $\left(\lambda_{0}, x_{0}\right) \in(0, \Lambda] \times(D(T) \cap D(C) \cap \partial \Omega)$ such that

$$
0 \in T x_{0}+C\left(\lambda_{0}, x_{0}\right)
$$


Proof (a) First, we prove that for every $s \in\left(0, s_{0}\right)$, there exists a $\left(\lambda_{0}, x_{0}\right) \in(0, \Lambda] \times(D(C) \cap$ $\partial \Omega$ ) such that

$$
T_{s} x_{0}+C\left(\lambda_{0}, x_{0}\right)+\varepsilon J_{\psi} x_{0}=0 .
$$

Assume on the contrary that for some $s \in\left(0, s_{0}\right)$ and for every $\lambda \in(0, \Lambda]$, the following holds:

$$
T_{s} x+C(\lambda, x)+\varepsilon J_{\psi} x \neq 0 \quad \text { for all } x \in D(C) \cap \partial \Omega \text {. }
$$

Then (3.2) holds for all $\lambda \in[0, \Lambda]$. For $\lambda=0$, the assertion is obvious.

For $t \in[0,1]$, we set $T^{t}=T_{s}$ and $C^{t}=C(t \Lambda, \cdot)+\varepsilon J_{\psi}$. To show that $\left\{T^{t}+C^{t}\right\}$ is an admissible homotopy in the sense of Definition 2.4 in [9], we have to check the following conditions on two families $\left\{T^{t}\right\}$ and $\left\{C^{t}\right\}$. In fact, conditions on $\left\{T^{t}\right\}$ are automatically satisfied, with $T^{t}$ independent of $t$, due to the monotonicity of $T_{s}$ and $T_{s}(0)=0$.

$\left(c_{1}^{t}\right)\left\{C^{t}\right\}$ is uniformly strongly quasibounded with respect to $T^{t}$, i.e., for every $\ell>0$ there exists a constant $K(\ell)>0$ such that for all $u \in L$ with $\|u\| \leq \ell$ and all $t \in[0,1]$,

$$
\left\langle T^{t} u+C^{t} u, u\right\rangle \leq 0 \quad \text { implies }\left\|C^{t} u\right\| \leq K(\ell) .
$$

This follows trivially from (c1) and the fact that $T_{s}$ and $J_{\psi}$ are monotone and $J_{\psi}$ is bounded.

$\left(c_{2}^{t}\right)$ For every pair of sequences $\left\{t_{j}\right\}$ in $[0,1]$ and $\left\{u_{j}\right\}$ in $L$ such that $t_{j} \rightarrow t_{0}, u_{j} \rightarrow u_{0}$, $C^{t_{j}} u_{j} \rightarrow h_{0}^{\prime \prime}$ and

$$
\limsup _{j \rightarrow \infty}\left\langle C^{t_{j}} u_{j}, u_{j}-u_{0}\right\rangle \leq 0, \quad\left\langle T^{t_{j}} u_{j}+C^{t_{j}} u_{j}, u_{j}\right\rangle \leq 0
$$

where $t_{0} \in[0,1], u_{0} \in X$, and $h_{0}^{*} \in X^{*}$, we have $u_{j} \rightarrow u_{0}, u_{0} \in D(C)$ and $C^{t_{0}} u_{0}=h_{0}^{*}$.

For this, there are two cases to consider. If $t_{0}=0$, then the second inequality in (3.3) implies

$$
\left.\varepsilon \psi\left(\left\|u_{j}\right\|\right)\left\|u_{j}\right\|=\varepsilon\langle\rangle_{\psi} u_{j}, u_{j}\right\rangle \leq\left\langle T_{s} u_{j}, u_{j}\right\rangle+\varepsilon\left\langle\bigcup_{\psi} u_{j}, u_{j}\right\rangle \leq-\left\langle C\left(t_{j} \Lambda, u_{j}\right), u_{j}\right\rangle \rightarrow 0
$$

and hence $u_{j} \rightarrow 0, u_{0}=0 \in D(C)$, and $C^{0} u_{0}=0=h_{0}^{*}$. Now let $t_{0}>0$. From the first inequality in (3.3) it follows that

$$
\left\langle C^{t_{j}} u_{j}, u_{j}-u_{0}\right\rangle \geq\left\langle C\left(t_{j} \Lambda, u_{j}\right), u_{j}-u_{0}\right\rangle+\varepsilon\left\langle J_{\psi} u_{0}, u_{j}-u_{0}\right\rangle
$$

and so

$$
\limsup _{j \rightarrow \infty}\left\langle C\left(t_{j} \Lambda, u_{j}\right), u_{j}-u_{0}\right\rangle \leq 0
$$

Combining this with

$$
\left\langle C\left(t_{j} \Lambda, u_{j}\right), u_{j}-u_{0}\right\rangle=\left\langle C\left(t_{j} \Lambda, u_{j}\right)-C\left(t_{0} \Lambda, u_{j}\right), u_{j}-u_{0}\right\rangle+\left\langle C\left(t_{0} \Lambda, u_{j}\right), u_{j}-u_{0}\right\rangle
$$


we have

$$
\begin{aligned}
\limsup _{j \rightarrow \infty}\left\langle C\left(t_{0} \Lambda, u_{j}\right), u_{j}-u_{0}\right\rangle & \leq \limsup _{j \rightarrow \infty}\left[-\left\langle C\left(t_{j} \Lambda, u_{j}\right)-C\left(t_{0} \Lambda, u_{j}\right), u_{j}-u_{0}\right\rangle\right] \\
& \leq \lim _{j \rightarrow \infty}\left\|C\left(t_{j} \Lambda, u_{j}\right)-C\left(t_{0} \Lambda, u_{j}\right)\right\|\left\|u_{j}-u_{0}\right\|=0 .
\end{aligned}
$$

In view of $C^{t_{j}} u_{j} \rightarrow h_{0}^{*}$, we can find a subsequence of $\left\{u_{j}\right\}$, denoted again by $\left\{u_{j}\right\}$, such that $C\left(t_{j} \Lambda, u_{j}\right) \rightarrow h_{1}^{*}$ and $J_{\psi} u_{j} \rightarrow h_{2}^{*}$ for some $h_{1}^{*}, h_{2}^{*} \in X^{*}$. Note that $C\left(t_{0} \Lambda, u_{j}\right) \rightarrow h_{1}^{*}$ and $h_{0}^{*}=$ $h_{1}^{*}+\varepsilon h_{2}^{*}$. Hence (c2) implies that $u_{j} \rightarrow u_{0}, u_{0} \in D(C)$, and $C\left(t_{0} \Lambda, u_{0}\right)=h_{1}^{*}$ and so $C^{t_{0}} u_{0}=$ $C\left(t_{0} \Lambda, u_{0}\right)+\varepsilon J_{\psi} u_{0}=h_{0}^{*}$. Thus, condition $\left(c_{2}^{t}\right)$ is satisfied in both cases.

$\left(c_{3}^{t}\right)$ For every $F \in \mathcal{F}(L)$ and $v \in L$, the function $\tilde{c}(F, v):[0,1] \times F \rightarrow \mathbb{R}, \tilde{c}(F, v)(t, u)=$ $\left\langle C^{t} u, v\right\rangle$, is continuous.

Actually, $\tilde{c}(F, v)$ is continuous on $[0,1] \times F$ because $c(t \Lambda, F, v)$ in the notation of (c3) is continuous on $F$ and $J_{\psi}$ is continuous on $X$. Consequently, we have shown that $\left\{T^{t}+C^{t}\right\}$, $t \in[0,1]$, is an admissible homotopy.

Following Kartsatos and Skrypnik [8,9], we can use the homotopy invariance property of the degree $d$ with respect to the bounded open set $\Omega$ as follows:

$$
\begin{aligned}
d\left(T_{s}+C(\lambda, \cdot)+\varepsilon J_{\psi}, \Omega, 0\right) & =d\left(T_{s}+\varepsilon J_{\psi}, \Omega, 0\right) \\
& =1
\end{aligned}
$$

for all $\lambda \in[0, \Lambda]$. The latter follows from Theorem 3 in [3], noticing that $T_{s}+\varepsilon J_{\psi}$ is demicontinuous, bounded, strictly monotone and satisfies condition $\left(S_{+}\right)$. For every $\lambda \in(0, \Lambda]$, the existence property of the degree implies that

$$
T_{s} x+C(\lambda, x)+\varepsilon J_{\psi} x=0 \quad \text { for some } x \in D(C) \cap \Omega \text {, }
$$

which contradicts property $(\mathcal{P})$. Therefore, the first assertion (3.1) is true.

According to assertion (3.1), let $\left\{s_{n}\right\}$ be a sequence in $\left(0, s_{0}\right)$ with $s_{n} \rightarrow 0$ and $\left\{\left(\lambda_{n}, x_{n}\right)\right\}$ be a sequence in $(0, \Lambda] \times(D(C) \cap \partial \Omega)$ such that

$$
T_{S_{n}} x_{n}+C\left(\lambda_{n}, x_{n}\right)+\varepsilon J_{\psi} x_{n}=0
$$

Without loss of generality, we may suppose that

$$
\lambda_{n} \rightarrow \lambda_{0}, \quad x_{n} \rightarrow x_{0}, \quad C\left(\lambda_{n}, x_{n}\right) \rightarrow c^{*} \quad \text { and } \quad J_{\psi} x_{n} \rightarrow j^{*},
$$

where $\lambda_{0} \in[0, \Lambda], x_{0} \in X, c^{*} \in X^{*}$ and $j^{*} \in X^{*}$. We may consider the case that $\lambda_{0} \in(0, \Lambda]$ as the conclusion of (a) does not hold for $\lambda_{0}=0$. In order to apply the condition $\left(\tilde{S}_{+}\right)$, we first show that

$$
\limsup _{n \rightarrow \infty}\left\langle C\left(\lambda_{n}, x_{n}\right), x_{n}-x_{0}\right\rangle \leq 0
$$

Assume on the contrary that there exists a subsequence of $\left\{x_{n}\right\}$, denoted again by $\left\{x_{n}\right\}$, such that

$$
\lim _{n \rightarrow \infty}\left\langle C\left(\lambda_{n}, x_{n}\right), x_{n}-x_{0}\right\rangle>0
$$


Note by (3.4) and the monotonicity of $J_{\psi}$ that

$$
\left\langle T_{s_{n}} x_{n}, x_{n}-x_{0}\right\rangle \leq-\left\langle C\left(\lambda_{n}, x_{n}\right), x_{n}-x_{0}\right\rangle-\varepsilon\left\langle J_{\psi} x_{0}, x_{n}-x_{0}\right\rangle
$$

and so

$$
\limsup _{n \rightarrow \infty}\left\langle T_{s_{n}} x_{n}, x_{n}-x_{0}\right\rangle<0
$$

Hence, it follows from $T_{s_{n}} x_{n} \rightarrow-c^{*}-\varepsilon j^{*}$ that

$$
\limsup _{n \rightarrow \infty}\left\langle T_{s_{n}} x_{n}, x_{n}\right\rangle<\left\langle-c^{*}-\varepsilon j^{*}, x_{0}\right\rangle
$$

For every $x \in D(T)$ and every $u^{*} \in T x$, it is shown as in the proof of part (a) of Theorem 2.1 that

$$
\liminf _{n \rightarrow \infty}\left\langle T_{s_{n}} x_{n}, x_{n}\right\rangle \geq\left\langle-c^{*}-\varepsilon j^{*}, x\right\rangle+\left\langle u^{*}, x_{0}-x\right\rangle
$$

which implies in view of (3.6)

$$
\left\langle-c^{*}-\varepsilon j^{*}-u^{*}, x_{0}-x\right\rangle>0 \text {. }
$$

Since $T$ is maximal monotone, we have $x_{0} \in D(T)$ and $-c^{*}-\varepsilon j^{*} \in T x_{0}$. Letting $x=x_{0}$ in (3.7), we have a contradiction. Thus, (3.5) holds.

As before, we can deduce from (3.5) that

$$
\limsup _{n \rightarrow \infty}\left\langle C\left(\lambda_{0}, x_{n}\right), x_{n}-x_{0}\right\rangle \leq 0 \text {. }
$$

Since $C$ satisfies condition $\left(\tilde{S}_{+}\right)$, we have

$$
x_{n} \rightarrow x_{0}, \quad x_{0} \in D(C), \quad \text { and } \quad C\left(\lambda_{0}, x_{0}\right)=c^{*} .
$$

Combining (3.4) and (3.8), we obtain

$$
T_{s_{n}} x_{n} \rightarrow-C\left(\lambda_{0}, x_{0}\right)-\varepsilon J_{\psi} x_{0}
$$

and

$$
J_{s_{n}} x_{n} \rightarrow x_{0} .
$$

Since $T$ is maximal monotone and $T_{s_{n}} x_{n} \in T J_{s_{n}} x_{n}$, Lemma 1.1 implies that $x_{0} \in D(T) \cap$ $D(C) \cap \partial \Omega$ and

$$
0 \in T x_{0}+C\left(\lambda_{0}, x_{0}\right)+\varepsilon J_{\psi} x_{0} .
$$


(b) In view of (a), for a sequence $\left\{\varepsilon_{n}\right\}$ in $\left(0, \varepsilon_{0}\right]$ with $\varepsilon_{n} \rightarrow 0$, we take sequences $\left\{\lambda_{n}\right\}$ in $(0, \Lambda],\left\{x_{n}\right\}$ in $D(T) \cap D(C) \cap \partial \Omega$ and $\left\{u_{n}^{*}\right\}$ in $X^{\prime \prime}$ with $u_{n}^{*} \in T x_{n}$ such that

$$
u_{n}^{*}+C\left(\lambda_{n}, x_{n}\right)+\varepsilon_{n} J_{\psi} x_{n}=0
$$

We may suppose that

$$
\lambda_{n} \rightarrow \lambda_{0}, \quad x_{n} \rightarrow x_{0}, \quad C\left(\lambda_{n}, x_{n}\right) \rightarrow c^{*} \quad \text { and } \quad J_{\psi} x_{n} \rightarrow j^{*},
$$

where $\lambda_{0} \in[0, \Lambda], x_{0} \in X, c^{*} \in X^{*}$ and $j^{*} \in X^{*}$. Note that $\lambda_{0} \in(0, \Lambda]$. As in the proof of part (b) of Theorem 2.1, a similar argument shows that

$$
\limsup _{n \rightarrow \infty}\left\langle C\left(\lambda_{n}, x_{n}\right), x_{n}-x_{0}\right\rangle \leq 0
$$

and so in a usual way

$$
\limsup _{n \rightarrow \infty}\left\langle C\left(\lambda_{0}, x_{n}\right), x_{n}-x_{0}\right\rangle \leq 0
$$

Hence, it follows from (c2) that

$$
x_{n} \rightarrow x_{0} \in \partial \Omega, \quad x_{0} \in D(C), \quad \text { and } \quad C\left(\lambda_{0}, x_{0}\right)=c^{\prime \prime}
$$

which implies by (3.9)

$$
u_{n}^{*} \rightarrow-C\left(\lambda_{0}, x_{0}\right) \text {. }
$$

Consequently, we obtain from the maximal monotonicity of $T$ that $x_{0} \in D(T)$ and

$$
0 \in T x_{0}+C\left(\lambda_{0}, x_{0}\right)
$$

This completes the proof.

Remark 3.2 In an analogous way to Theorem 3.1, we can observe the eigenvalue problem $0 \in T x+\lambda C x$ for quasibounded perturbations of maximal monotone operators; see [10].

\section{Competing interests}

The author declares that she has no competing interests.

\section{Acknowledgements}

This research was supported by Basic Science Research Program through the National Research Foundation of Korea (NRF) funded by the Ministry of Education, Science and Technology (NRF-2011-0021-829).

Received: 24 March 2012 Accepted: 3 October 2012 Published: 17 October 2012

\section{References}

1. Brezis, H, Crandall, MG, Pazy, A: Perturbations of nonlinear maximal monotone sets in Banach space. Commun. Pure Appl. Math. 23, 123-144 (1970)

2. Browder, FE: Fixed point theory and nonlinear problems. Bull. Am. Math. Soc. 9, 1-39 (1983)

3. Browder, FE: Degree of mapping for nonlinear mappings of monotone type. Proc. Natl. Acad. Sci. USA 80, 1771-1773 (1983) 
4. Browder, FE: The degree of mapping and its generalizations. Contemp. Math. 21, 15-40 (1983)

5. Guan, Z, Kartsatos, AG: On the eigenvalue problem for perturbations of nonlinear accretive and monotone operators in Banach spaces. Nonlinear Anal. 27, 125-141 (1996)

6. Kartsatos, AG: New results in the perturbation theory of maximal monotone and $m$-accretive operators in Banach spaces. Trans. Am. Math. Soc. 348, 1663-1707 (1996)

7. Kartsatos, AG, Skrypnik, IV: Normalized eigenvectors for nonlinear abstract and elliptic operators. J. Differ. Equ. 155 , 443-475 (1999)

8. Kartsatos, AG, Skrypnik, IV: Topological degree theories for densely defined mappings involving operators of type $\left(S_{+}\right)$. Adv. Differ. Equ. 4, 413-456 (1999)

9. Kartsatos, AG, Skrypnik, IV: A new topological degree theory for densely defined quasibounded $\left(\tilde{S}_{+}\right)$-perturbations of multivalued maximal monotone operators in reflexive Banach spaces. Abstr. Appl. Anal. 2005, 121-158 (2005)

10. Kartsatos, AG, Skrypnik, IV: On the eigenvalue problem for perturbed nonlinear maximal monotone operators in reflexive Banach spaces. Trans. Am. Math. Soc. 358, 3851-3881 (2006)

11. Kim, I-S: Some eigenvalue results for maximal monotone operators. Nonlinear Anal. 74, 6041-6049 (2011)

12. $\mathrm{Li}, \mathrm{H}-\mathrm{X}$, Huang, F-L: On the nonlinear eigenvalue problem for perturbations of monotone and accretive operators in Banach spaces. Sichuan Daxue Xuebao 37, 303-309 (2000)

13. Petryshyn, WV: Approximation-Solvability of Nonlinear Functional and Differential Equations. Marcel Dekker, New York (1993)

14. Skrypnik, IV: Nonlinear Higher Order Elliptic Equations. Naukova Dumka, Kiev (1973) (Russian)

15. Skrypnik, IV: Methods for Analysis of Nonlinear Elliptic Boundary Value Problems. Transl., Ser. II., vol. 139. Am. Math. Soc., Providence (1994)

16. Väth, M: Fixed point theorems and fixed point index for countably condensing maps. Topol. Methods Nonlinear Anal. 13, 341-363 (1999)

17. Zeidler, E: Nonlinear Functional Analysis and Its Applications II/B: Nonlinear Monotone Operators. Springer, New York (1990)

doi:10.1186/1687-1812-2012-178

Cite this article as: Kim: Implicit eigenvalue problems for maximal monotone operators. Fixed Point Theory and Applications 2012 2012:178.

\section{Submit your manuscript to a SpringerOpen ${ }^{\circ}$ journal and benefit from:}

- Convenient online submission

Rigorous peer review

- Immediate publication on acceptance

- Open access: articles freely available online

- High visibility within the field

- Retaining the copyright to your article 